\section{Avaliação do Programa de Controle da Doença de Chagas em relação à presença de Panstrongylus megistus na região centro-oeste do Estado de Minas Gerais, Brasil}

\author{
Evaluation of the Chagas Disease Control Program \\ and presence of Panstrongylus megistus in central- \\ western Minas Gerais State, Brazil
}

\author{
${ }_{1}$ Centro de Pesquisas René \\ Rachou, Fundação Oswaldo \\ Cruz, Belo Horizonte, Brasil. \\ 2 Gerência Regional de Saúde \\ de Divinópolis, Divinópolis, \\ Brasil. \\ Correspondência \\ J. C. P. Dias \\ Centro de Pesquisas René \\ Rachou, Fundação Oswaldo \\ Cruz. \\ C. P. 1743, Belo Horizonte, $M G$ \\ 30190-012, Brasil. \\ jcpdias@cpqrr.fiocruz.br
}

\begin{abstract}
The Brazilian National Chagas Disease Control Program (PCDCh) is currently in the epidemiological surveillance phase and poses one of the principal challenges for maintaining and improving surveillance. With the decentralization of health actions, leading to the transfer of responsibilities over the control of endemic diseases to the State and Municipal governments, some municipalities have faced difficulties in implementing the Program, since they lack experience in controlling this disease. This study analyzes the current situation with epidemiological surveillance of Chagas disease in 54 municipalities (counties) in central-western Minas Gerais State, with a view towards evaluation and improvement of the process. From July 2003 to July 2007, 776 captures were performed, totaling 1,390 triatomine bugs from 37 municipalities. The most prevalent species was Panstrongylus megistus (99.3\% of the specimens captured). A total of $8.3 \%$ of the specimens were positive for Trypanosoma cruzi-like flagellates. Distribution of the specimens differed between municipalities, and the article discusses some reasons for these differences. As a whole, the findings strongly suggest the need to improve Chagas disease epidemiological surveillance in the region.
\end{abstract}

Chagas Disease; Communicable Disease Control; Epidemiologic Surveillance
Marcos Marreiro Villela ${ }^{1}$

Janice Maria Borba de Souza 2

Vicente de Paula Melo 2

João Carlos Pinto Dias 1

\section{Introdução}

No Brasil, a doença de Chagas humana incide em praticamente todos os estados, sendo sua ocorrência dependente basicamente da distribuição dos vetores domiciliados. Visto que não existe vacina que evite a infecção da doença, o modo de se impedir o aparecimento de novos casos reside, especialmente, no controle dos principais mecanismos de transmissão (vetorial, transfusional, congênita e, atualmente, oral) do protozoário Trypanosoma cruzi ${ }^{1}$. Como a imensa maioria dos chagásicos existentes foi contaminada pelas fezes dos triatomíneos infectados, o afastamento destes hemípteros da moradia deve ser o enfoque preventivo principal 2 .

As ações de controle da transmissão vetorial da tripanossomíase americana no Brasil começaram a se esboçar a partir da década de 1940 , objetivando diminuir a transmissão da doença no ambiente domiciliar. O Programa de Controle da Doença de Chagas (PCDCh) foi institucionalizado em 1950 pelo Serviço Nacional de Malária, mas só foi sistematizado e estruturado na forma de programa de alcance nacional em 1975 pela Superintendência de Campanhas de Saúde Pública (SUCAM), atual Fundação Nacional de Saúde (FUNASA) 3,4.

No ano de 1983, o PCDCh foi priorizado e recebeu do Governo Federal um recurso em torno de 15 milhões de dólares, quantia suficiente para cobrir toda a área endêmica, representada por 
2.450 municípios, onde se realizaram mais de 700 mil expurgos domiciliares, sendo o controle vetorial exercido em caráter pleno 5,6. As ações de controle adotadas tiveram êxito e se obteve a eliminação do principal vetor (Triatoma infestans) de amplas áreas do Brasil, bem como a redução da infestação e densidade intradomiciliar das demais espécies responsáveis pela transmissão domiciliar do T. cruzi 7,8. Os índices de transmissão vetorial declinaram drasticamente em mais de 95\% da área endêmica. Além disso, a transmissão transfusional também sofreu enorme redução a partir do final da década de 1980, principalmente em virtude da ameaça da AIDS, que veio a melhorar sensivelmente o controle e a qualidade das transfusões de sangue no país 9,10. Grandes avanços também foram obtidos em outros países latino-americanos, sobretudo a partir da Iniciativa dos Países do Cone Sul, criada em 1991, a qual objetivou a eliminação de T. infestans dos domicílios e a triagem dos doadores de sangue dos países envolvidos (Argentina, Bolívia, Brasil, Chile, Paraguai e Uruguai) 11,12. Somandose as medidas preventivas a outros fatos (como o êxodo rural), reduziu-se o número de pessoas vivendo em áreas de risco para aquisição de $T$. cruzi, de 100 milhões para aproximadamente 40 milhões de pessoas na América Latina 13

Em decorrência da descentralização do setor saúde, que teve grande impulso no início da década de 1990, um fato importante ocorreu com o PCDCh em 1999, a partir da Portaria $n^{\circ} .1 .399$ do Ministério da Saúde ${ }^{14}$, que equacionou a descentralização do sistema de saúde e reduziu as ações da FUNASA, promovendo a transferência das responsabilidades das ações de epidemiologia e de controle das doenças para os estados e municípios 15. Em alguns estados se formaram as Gerências Regionais de Saúde (GRS), que passaram a coordenar e supervisionar as ações realizadas pelas entidades municipais. Em Minas Gerais, estas GRS pertencem à Secretaria Estadual de Saúde de Minas Gerais (SES-MG), e estão sediadas em municípios de maior importância política/econômica, existindo hoje 28 GRS no estado (Secretaria do Estado da Saúde de Minas Gerais. http://www.saude.mg.gov.br/institucio nal/grs, acessado em 25/Mar/2008).

Em 2004, a Portaria $n^{\circ} .1 .399$ sofreu ligeiros ajustes que resultaram na Portaria $n^{\circ} .1 .172$ 16, a qual estabeleceu melhor o processo de programação e acompanhamento das ações de saúde e definiu a certificação municipal, a sistemática do financiamento destinado às endemias, assim como as penalidades para o descumprimento das responsabilidades. Para receber a certificação de gestão e assumir as ações de saúde, o município precisa comprovar capacidade técnico-operativa e dispor de Conselho Municipal e Fundo Municipal de Saúde como principais exigências. A quantidade de recurso para cada município depende de sua situação epidemiológica, volume das operações, extensão territorial e curso histórico de suas atividades. Um instrumento de controle das aplicações financeiras, previsto na Portaria $n^{\circ}$. 11.72, estabelece a suspensão da transferência mensal dos recursos para os estados e municípios que não fizerem a demonstração a contento da aplicação dos recursos.

Em seu marco teórico a descentralização se mostra como importante caminho, beneficiando diretamente a população, dando-lhe rapidez, integralidade e eficiência nas respostas quanto à vigilância epidemiológica das endemias, além de maior acesso a saúde, ao passo que desde o final da década de 1970, vêm se multiplicando os centros e postos de saúde no Brasil 17. Também permitiria, teoricamente, melhor controle social e visão epidemiológica mais específica ao município. Uma das dificuldades deste novo sistema é que embora a descentralização se justifique em pressupostos teóricos, não existe tradição no controle das endemias pelos municípios, o que dificulta a transferência de encargos e a realização de programas que competem com as ações tradicionais de atenção à saúde nesse nível, além de, praticamente, desmantelar os serviços de controle de instituições tradicionais como a FUNASA 18

Como uma conseqüência do sucesso na eliminação de T. infestans no Brasil, celebrada em 2006, o PCDCh tem enfrentado dificuldades para manutenção em vários locais do país. Por vezes existe a incorporação da idéia de que a eliminação do principal vetor representa a erradicação ou o controle efetivo da doença de Chagas, esquecendo-se da importância epidemiológica de outras espécies nativas e da necessidade de manutenção da correspondente vigilância epidemiológica 19

O presente trabalho objetivou avaliar a situação atual da vigilância entomológica da doença de Chagas em 54 municípios da região centrooeste de Minas Gerais, após a descentralização da saúde, com vistas ao aprimoramento do processo.

\section{Materiais e métodos}

\section{Escolha da área}

A região escolhida trata-se de um local onde a doença de Chagas foi freqüente no passado, existindo importante cunho histórico na região. Este estudo abrangeu todos os 54 municípios coorde- 
nados pela GRS-Divinópolis. O mapa da região pode ser visualizado em artigos anteriores, publicados neste mesmo periódico por nosso grupo de pesquisa 14,20 . Esta GRS foi escolhida por estar estruturada e em funcionamento, dispondo-se de bons dados históricos sobre a epidemiologia da doença, além de contar com a participação e presença de antigos funcionários da FUNASA, totalmente solidários e colaboradores para com a pesquisa.

\section{Acompanhamento e análise da vigilância entomológica}

Nos 54 municípios selecionados, os triatomíneos foram capturados seguindo basicamente as normativas clássicas da FUNASA 21 e aprimoradas pela Gerência Técnica em Doença de Chagas da SES-MG. Os insetos foram capturados no período de julho de 2003 até março de 2007 por três vias:

1) Notificação de triatomíneos: realizada pela própria população, que leva o inseto suspeito até um Posto de Informação de Triatomíneo (PIT), instalado em locais estratégicos dos municípios, como escolas e postos de saúde;

2) Atendimento à notificação: procedida pelos agentes que trabalham no PCDCh de cada município;

3) Pesquisa ativa, na qual é realizada a procura de vetores em todas as unidades domiciliares do município, independente de a população ter realizado ou não alguma notificação. As unidades domiciliares são constituídas pela casa e seus anexos (galinheiro, casa de despejo etc.), os quais consideram-se peridomicílio.

Os moradores são orientados pelos agentes do PCDCh sobre os prováveis locais de encontro dos insetos, e instruídos a capturá-los com o devido cuidado e enviá-los para o PIT mais próximo da sua residência. Os insetos capturados nos municípios foram enviados para Divinópolis juntamente com uma ficha padronizada específica para a presente pesquisa, com algumas informações extras sobre a captura, sobretudo contemplando características do peridomicílio. Na tentativa de uniformizar as capturas, foram realizadas reuniões com todos os agentes municipais antes de se principiar a pesquisa, por meio das quais se explicaram o propósito e os objetivos da mesma. Ao final do estudo foi realizado um encontro sobre o PCDCh no Município de Pimenta, que contou com a participação dos agentes e secretários de saúde municipais, sendo proferidas palestras sobre resultados desta pesquisa e os meios de aprimorar o PCDCh dos municípios.

\section{Resultados}

De julho de 2003 até março de 2007, 37 (68,5\%) municípios enviaram fichas referentes ao estudo. Foram capturadas quatro espécies de triatomíneos, sendo Panstrongylus megistus a espécie mais freqüente, representando 99,3\% (1.380) dos insetos capturados, seguido de Panstrongylus diasi (5 ou $0,4 \%$ ), Triatoma sordida (3 ou 0,2\%) e Rhodnius neglectus (2 ou 0,1\%). P. megistus ocorreu em todos os 37 municípios, $P$. diasi foi capturado em Itatiaiuçu, Martinho Campos e Pitangui, T. sordida foi capturado em Pains e $R$. neglectus em Bambuí e São Sebastião do Oeste.

No decorrer do período de quatro anos, foram totalizadas 776 capturas, sendo que o maior número destas ocorreu no período de julho de 2005 até junho de 2006, sendo este período coincidente à maior quantidade de triatomíneos capturados (Tabela 1). A maioria das capturas compreendeu apenas um triatomíneo (531 ou $68,4 \%$ das vezes).

Tabela 1

Quantidade de capturas e número total de triatomíneos capturados no período de julho de 2003 até junho de 2007, em municípios da região centro-oeste de Minas Gerais, Brasil.

\begin{tabular}{|c|c|c|c|c|}
\hline \multirow[t]{2}{*}{ Período * } & \multicolumn{2}{|c|}{ Quantidade de capturas } & \multicolumn{2}{|c|}{ Quantidade de triatomíneos } \\
\hline & $\mathbf{n}$ & $\%$ & $\mathbf{n}$ & $\%$ \\
\hline $2003 / 2004$ & 132 & 17 & 255 & 18,3 \\
\hline $2004 / 2005$ & 146 & 18,8 & 233 & 16,8 \\
\hline $2005 / 2006$ & 267 & 34,4 & 464 & 33,4 \\
\hline $2006 / 2007$ & 231 & 29,8 & 438 & 31,5 \\
\hline Total & 776 & 100,0 & 1.390 & 100,0 \\
\hline
\end{tabular}

* O período compreende de julho de um ano até junho do ano seguinte. 
As 776 capturas de triatomíneos foram distribuídas em 433 notificações (55,8\%), 201 atendimentos (25,9\%), e 142 por pesquisa ativa (18,3\%). Embora o maior número de capturas ocorra nas notificações $(55,8 \%)$, a maior quantidade de insetos é capturada durante os atendimentos, somados à pesquisa ativa (Tabela 2).

Quando analisada a quantidade de capturas ocorridas no intradomicílio e peridomicílio, notou-se que o maior número ocorreu no intradomicílio, sendo a notificação a principal responsável pelas capturas neste ambiente. No peridomicílio o maior número de capturas deu-se no atendimento, pelos agentes municipais. Não houve diferença significativa no número de capturas do intra e peridomicílio ( $\mathrm{p}>0,05)$ (Tabela 3$)$.

A maior quantidade de triatomíneos foi capturada no peridomicílio (844 insetos), durante o atendimento (334 ou $39,6 \%$ das oportunidades) e a pesquisa ativa (335 ou 39,7\% das oportunidades), sendo esta diferença significativa em relação à quantidade de insetos provenientes do intradomicílio (546 insetos), os quais são apreendidos especialmente durante a notificação $(65,6 \%)(\mathrm{p}<0,05)$.

No intradomicílio, o quarto $(58,1 \%)$, a sala $(22,6 \%)$ e a cozinha $(13,2 \%)$ foram os ambientes que propiciaram o maior número de capturas de triatomíneos, enquanto que no peridomicílio as capturas foram mais freqüentes no galinheiro $(66,2 \%)$, seguindo-se do paiol $(15,3 \%)$ e casa de despejo (7,5\%)

No que tange ao estádio evolutivo dos insetos, o mais capturado foi o adulto (50,8\%), seguido de ninfa de quinto estádio $(20,8 \%)$, quarto estádio $(9,9 \%)$, terceiro estádio $(9,7 \%)$, segundo estádio (6,3\%), e primeiro estádio (2,5\%). Associando-se o estádio evolutivo com as formas de captura, a notificação foi responsável por $52 \%$ das capturas dos insetos adultos, e a pesquisa ativa por $17 \%$, diferentemente do que ocorre para as ninfas de primeiro estádio, pois $60 \%$ dos seus encontros se deram durante a pesquisa ativa, e apenas 14,3\% destes insetos foram capturados através da população (notificação) (Figura 1)

Quanto à colonização (presença de ninfas num determinado ecótopo), esta ocorreu em $41,4 \%$ das capturas (321 oportunidades), destas, $108(33,6 \%)$ ocorreram no intradomicílio e $213(66,4 \%)$ no peridomicílio. Considerando-se apenas os domicílios com colonização intradomiciliar, o quarto foi o cômodo com maior índice $(66,7 \%)$, seguido da cozinha (12\%) e da sala $(10,2 \%)$. Fora da casa, o galinheiro foi o anexo com maior número de colônias de triatomíneos $(67,6 \%)$, seguido do paiol (12,7\%), e casa de despejo $(5,6 \%)$.

Tabela 2

Porcentagem das capturas de triatomíneos de acordo com a forma de captura e quantidade de triatomíneos capturados em cada uma delas, entre julho de 2003 e junho de 2007, na região centro-oeste de Minas Gerais, Brasil.

\begin{tabular}{lccccccc}
\hline Capturas & Notificação & $\%$ & Atendimento & $\%$ & Pesquisa ativa & $\%$ & Total \\
\hline Quantidade de capturas & 433 & 55,8 & 201 & 25,9 & 142 & 18,3 \\
Quantidade de triatomíneos & 533 & 38,3 & 452 & 32,5 & 405 & 29,1 & 1.390 \\
\hline
\end{tabular}

Tabela 3

Distribuição da quantidade e das formas de capturas de triatomíneos em relação ao intra e ao peridomicílio, de insetos provenientes da região centro-oeste de Minas Gerais, Brasil, entre julho de 2003 até junho de 2007.

\begin{tabular}{lcccc}
\hline Capturas & & \multicolumn{2}{c}{ Quantidade de capturas } \\
& Intradomicílio & $\%$ & Peridomicílio & $\%$ \\
\hline Notificação & 309 & 77,8 & 124 & 32,7 \\
Atendimento & 58 & 14,6 & 143 & 37,7 \\
Pesquisa ativa & 30 & 7,6 & 112 & 29,6 \\
Total & 397 & 100,0 & 379 & 100,0 \\
\hline
\end{tabular}


Distribuição dos estádios evolutivos de acordo com a forma de captura dos triatomíneos provenientes da região centro-oeste de Minas Gerais, Brasil, entre julho de 2003 e junho de 2007 ( $n=1.390$ ).

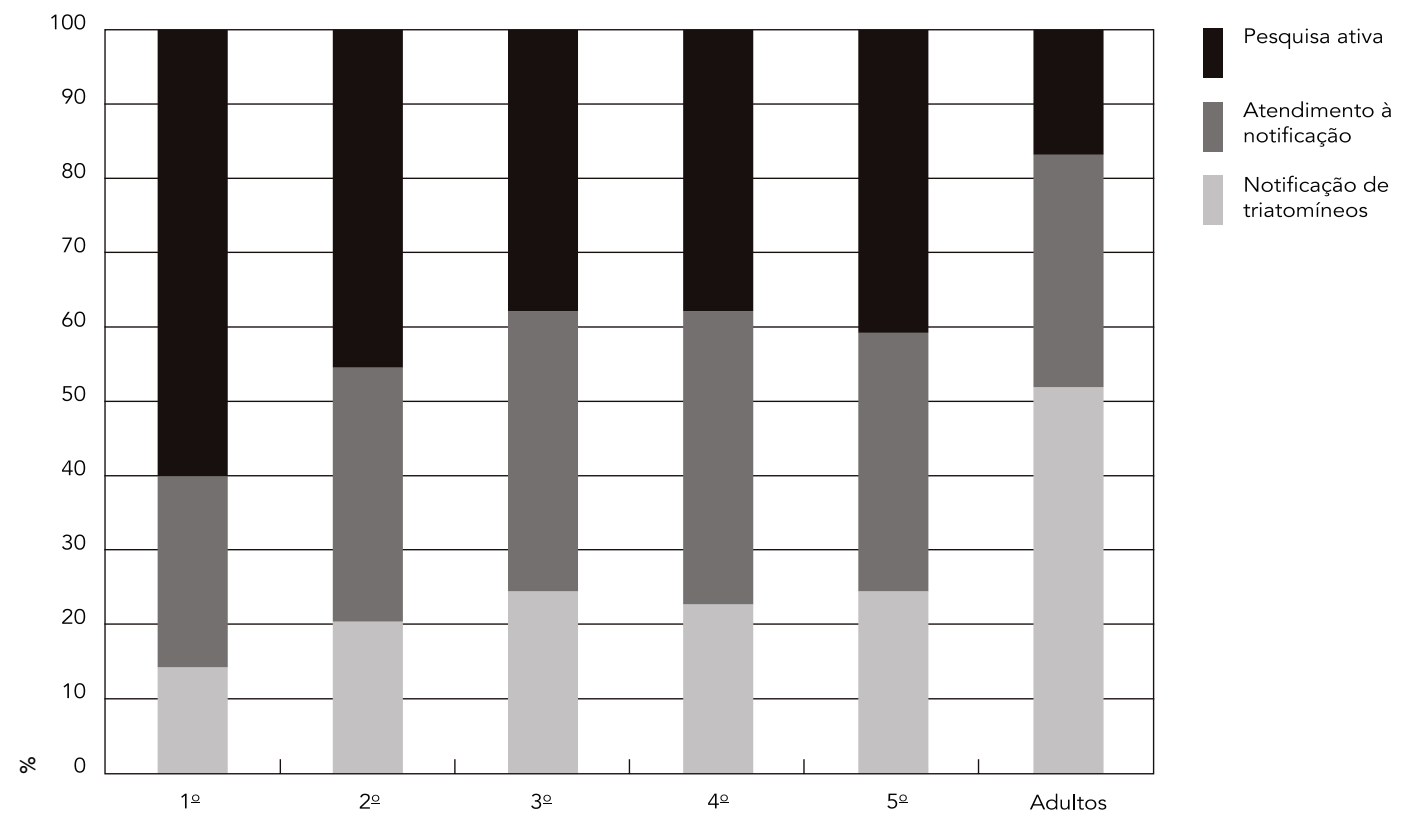

O índice de positividade para flagelados semelhantes ao T. cruzi dos triatomíneos capturados foi de $8,3 \%$ (115 insetos entre 1.386 examinados), sendo maior entre os adultos $(9,9 \%)$, do que em ninfas $(6,6 \%)$. Somente a partir do terceiro estádio de desenvolvimento foram diagnosticados insetos positivos para flagelados semelhantes ao T. cruzi.

A distribuição das capturas nos municípios mostrou diferenças entre as entidades municipais. O maior número de capturas ocorreu em Piracema (136 capturas), seguido de Bambuí (100 capturas), Itaguara (47 capturas) e Itatiaiuçu (41 capturas). Em 17 municipalidades nenhuma ficha foi enviada (Araújos, Arcos, Bom Despacho, Conceição do Pará, Córrego Fundo, Divinópolis, Igaratinga, Japaraíba, Lagoa da Prata, Moema, Onça do Pitangui, Perdigão, Sant'ana do Jacaré, Santo Antônio do Monte, São Gonçalo do Pará, São José da Varginha e Serra da Saudade).

Quanto ao aspecto físico das unidades domiciliares analisadas e seu entorno, das 334 casas avaliadas pelos funcionários durante o atendimento, ou pesquisa ativa, notou-se que 266 $(79,6 \%)$ unidades domiciliares se constituíam de alvenaria boa, 36 (10,8\%) de alvenaria ruim, e 32
(9,9\%) eram de barro/adobe, sendo que 20 delas foram consideradas de más condições.

Também foram reportados aspectos do peridomicílio em 334 unidades domiciliareso, nas quais se capturaram triatomíneos, considerando-se uma distância de até 200m: em 85,9\% (277) das vezes havia capões de mato nos arredores; existiam casas próximas (vizinhas) em 77,5\% (259) das oportunidades; e palmeiras se fizeram presentes em 54,5\% (175) das ocasiões, sendo que a maioria das palmeiras $(94,9 \%)$, tratava-se de "macaúbeiras" (Acrocomia aculeata).

\section{Discussão e conclusões}

Segundo Vinhas \& Schofield ${ }^{22}$, toda a campanha de controle de doenças apresenta sua maior dificuldade próxima do final, como sendo um “castigo pelo sucesso". Deste modo é de extrema relevância que se realizem avaliações nesta etapa de vigilância, com o intuito de diagnosticar problemas e propor soluções para os programas.

Este estudo faz parte de um acompanhamento do PCDCh realizado pela Fundação Oswaldo Cruz (FIOCRUZ) de Minas Gerais, sen- 
do uma das primeiras abordagens sobre o funcionamento do programa na nova conjuntura administrativa 15,20,23.

Dos 54 municípios que participaram da pesquisa, 37 enviaram fichas referentes à pesquisa. A ausência de participação dos outros municípios pode explicar-se pela falta de captura por parte do agente municipal em virtude de inexperiência ou práticas pouco eficientes do funcionário; ausência de insetos invadindo o domicílio nestas localidades; desativação do PCDCh em termos estruturais; ou mesmo a simples falta de registro junto à GRS. Todas estas possibilidades são passíveis de ocorrer, visto que várias dificuldades foram apontadas tanto por agentes municipais como pelos técnicos da GRS. Em boa parte dos casos, a maior quantidade de triatomíneos capturada em algum município se associa à experiência, à estabilidade e à competência do agente municipal, como também à maior densidade de vetores numa determinada região. Como exemplo se pode citar o Município de Piracema, que teve o maior número de capturas registradas no período. Neste município, através de entrevistas realizadas com o agente e por meio de observações feitas pelos coordenadores da GRS, tornaram-se evidentes a dedicação e o conhecimento do funcionário para a atividade, assim como o apoio prestado pelos gestores municipais sempre que o agente necessitava de algum auxílio para exercer o cargo. Sendo assim, seguidamente o Município de Piracema foi solicitado a sediar cursos de capacitação aos agentes recém-contratados de municípios vizinhos. Piracema também se tratou de um dos poucos municípios a não trocar de agente em todo o período desta análise. Algumas cidades, como Paíns, Perdigão e Dores do Indaiá, trocaram de funcionários diversas vezes, denotando alta rotatividade dos mesmos por diversos fatores, o que resultou na fragmentação e desativação do PCDCh nestes locais.

Segundo Silveira 24, a descentralização pode originar benefícios aos programas de controle de endemias, como: respostas mais oportunas e eficazes, em virtude da possibilidade de efetuar intervenções imediatas; maior adequação das ações em razão de os funcionários municipais apresentarem conhecimento preciso da realidade local; assim como a maior continuidade das operações nas municipalidades devido à possibilidade de serem executadas pelos serviços locais de saúde. Porém o mesmo autor relatou algumas dificuldades capazes de ocorrer no novo sistema: falta de prioridade por parte das autoridades locais, além da perda de coesão das ações, com risco de que as mesmas se desenvolvam de forma desarticulada e descontinuada. Esses problemas foram diagnosticados no pre- sente trabalho e em outros anteriores efetuados na mesma região 20 .

Cerqueira et al. 25 relataram que o processo de descentralização das endemias no Brasil não ocorreu de forma gradativa, não tendo havido preparação adequada de repasse das responsabilidades da vigilância epidemiológica para os municípios. Em nossa análise, é particularmente preocupante a situação de municípios com histórico de presença de P. megistus nos domicílios, em que as intervenções são mínimas ou ausentes. Outras informações relativas à discussão desta supervisão nos municípios e avaliação dos agentes municipalizados podem ser vistas em outro estudo por nós realizado, por meio do qual ficaram registradas inúmeras carências e dificuldades que os agentes municipais desta região apresentam na execução do programa 20 .

A espécie mais detectada tanto no ambiente domiciliar como no peridomiciliar foi P. megistus. O presente estudo corrobora citações que afirmam que eliminado-se T. infestans de Minas Gerais, $P$. megistus voltou a constituir-se no vetor mais importante da doença de Chagas no estado, sendo a espécie mais capturada no âmbito domiciliar 26,27. O encontro de ninfas de P. megistus em $41,4 \%$ das capturas evidencia o processo de domiciliação da espécie, e confirma os achados já encontrados para a mesma região 15,28 . Verificouse que a domiciliação ocorreu principalmente no peridomicílio $(66,4 \%)$, ambiente problemático para o controle químico dos insetos, uma vez que, neste local, o efeito residual dos inseticidas é menor, pois os mesmos sofrem maior ação das variações climáticas, como a incidência direta do sol, chuvas etc. 29,30 .

Das unidades domiciliares em que se anotaram alguns aspectos relativos ao redor da casa, percebeu-se que a maioria apresentava casas próximas, capões de mato e/ou palmeiras, até uma distância de $200 \mathrm{~m}$, sendo que todos estes ambientes podem servir de fonte de infestação de triatomíneos para os domicílios. No Estado de São Paulo, quando se ampliou o raio de pesquisa para as casas vizinhas àquela notificante, se obteve resultado expressivo, apontando que as mesmas devem ser pesquisadas, pois há maiores chances do encontro de novos focos de triatomíneos 31. Quanto às palmeiras, estas apresentam grande relevância ecológica e epidemiológica para os vetores da doença de Chagas, em virtude de estabelecerem biótopo que pode oferecer esconderijo e moradia a pássaros, roedores e marsupiais, os quais comumente servem de fonte alimentar para os hemípteros hematófagos, também constituindo-se alguns desses animais em fonte de infecção por T. cruzi 32,33. A presença de capões de mato próximos 
aos domicílios também possibilita que triatomíneos partam destes locais para dentro das unidades domiciliares. Uma das medidas que pode ser adotada no controle dos insetos é o manejo correto do peridomicílio, e, se possível, construí-los em locais distantes de fontes de infestação de triatomíneos, como palmeiras e capões de mato.

Através da avaliação das casas, observou-se que $10,8 \%$ dos domicílios são de alvenaria ruim e 9,9\% são de barro/adobe, sendo várias casas consideradas em más condições, possibilitando múltiplos esconderijos para os "barbeiros". Este é um problema comum em vários países latinoamericanos, como conseqüência da miserável situação econômica e da instabilidade social em que muitas famílias vivem ${ }^{34}$. Não obstante, chama atenção o fato de $79,6 \%$ das habitações encontradas infestadas serem de boa alvenaria, indicando pressão de domiciliação sobre ecótopos artificiais melhor organizados. Evidencia-se aqui uma complexidade maior na questão epidemiológica, relativizando-se de certa forma o papel da boa habitação em áreas de vigilância sobre espécies triatomínicas ubiquistas, em áreas de peridomicílio infestado e com focos silvestres em seu entorno, situações essas já consideradas para Triatoma vitticeps 35 , Triatoma brasiliensis $36 \mathrm{e}$ mesmo para $P$. megistus 37 .

Para o peridomicílio o maior número de capturas realizou-se pelos agentes municipais, fato também encontrado no Estado de São Paulo 38. Este episódio pode ser explicado em decorrência do melhor preparo do agente para diagnosticar os possíveis focos presentes no peridomicílio, onde os esconderijos dos insetos são usualmente mais numerosos, e a população está desatenta à presença de triatomíneos, além de ter menor cuidado com a higiene e organização deste local. Uma pesquisa realizada no Estado do Paraná revelou o descaso dos moradores com o peridomicílio, que independente das condições de cuidado do domicílio, era deixado em abandono, muitas vezes porque a própria população não percebia tal ambiente como fazendo parte da moradia 39 . Aqui é fortemente demonstrada a importância da manutenção de um programa sistematizado e regular, visto que a maioria dos insetos do peridomicílio é somente detectada pelos agentes municipais.

Outras espécies de triatomíneos encontradas na região foram $P$. diasi, T. sordida e $R$. neglectus. Destas espécies, todos os insetos capturados eram adultos e nenhum estava positivo para flagelados semelhantes ao T. cruzi, o que sugere sua origem silvestre, baixo poder de colonização e associação básica com aves ou outras fontes não infectadas pelo T. cruzi ${ }^{40,41}$.
Cabe destacar que é realizada ampla devastação do ambiente silvestre formação de pastagens e, mais recentemente, para a cultura de cana-deaçúcar em alguns municípios da região centrooeste de Minas Gerais, objetivando estimular a produção de álcool com vistas à obtenção de energia alternativa. Esta modificação/degradação do ambiente natural certamente vem reduzindo a fauna silvestre primitiva, assim contribuindo para a redução de insetos capturados, por exemplo, no Município de Bambuí 28. Não obstante, no decorrer dos quatro anos da corrente pesquisa, percebeu-se que nos últimos dois anos (2005/2006, 2006/2007) houve aumento significativo no número de capturas, o que refletiu na maior quantidade de insetos apanhados. Ao que tudo indica tal diferença não deve ser interpretada como tendência crescente de domiciliação dos insetos, e sim como uma maior abrangência e/ou melhoria dos serviços realizados pelos agentes do PCDCh, como já denotado em outras regiões 42 . A partir de reuniões realizadas nos últimos meses de pesquisa na GRS-Divinópolis, se evidenciou que o número de agentes efetivados pelas prefeituras aumentou nos últimos anos, e com isso a rotatividade dos mesmos diminuiu, resultando em funcionários mais experientes e comprometidos com o serviço, daí resultando um maior número de capturas.

Considerando-se o estádio evolutivo em que os insetos são capturados, nota-se que quanto maior seu tamanho, os mesmos são mais facilmente apreendidos, tanto pela população quanto pelos agentes. Isto sugere que a população, e mesmo os agentes, estão mais sensibilizados (ou preparados) para a captura de insetos adultos, tanto pela maior facilidade de encontro destes devido a seu tamanho, como pelo conhecimento e maior divulgação deste estádio evolutivo. Este fato, que se repete em outras áreas como São Paulo e Góias é importante para o aprimoramento da vigilância epidemiológica, devendo ser levado em consideração nos trabalhos de natureza educativa 43

O índice de infecção para flagelados semelhantes ao T. cruzi dos triatomíneos capturados foi de $8,3 \%$ sendo maior nos adultos do que nas ninfas, o que é encontrado também em outras observações, uma vez que os insetos adultos realizam repastos por muito mais vezes que as ninfas 44. A taxa de infecção por nós constatada foi inferior à encontrada no Município de Uberaba (Minas Gerais) e na região de Campinas (São Paulo), onde a espécie (também P. megistus) acusou índice de infecção de 26,6\% e 10,26\%, respectivamente, tendo sido associadas estas altas taxas ao grande número de didelfídeos e ratos vistos na região 45,46. Entretanto, a infecção diagnosticada 
neste trabalho foi superior à diagnosticada em Bambuí na década de 1990 47, na qual os autores concluíram que o baixo índice de infecção se devia à alta proporção de sangue de aves diagnosticado na reação de precipitina. Cabe frisar que estudos sobre a fonte (origem) alimentar dos triatomíneos também são realizadas na presente pesquisa, e os resultados serão objetivados em outras publicações. Também é importante informar que os exames sorológicos de rotina realizados na região apontam ausência de transmissão vetorial da doença.

Conforme os dados apresentados, foram percebidos riscos para a manutenção do controle da doença de Chagas, sendo um dos principais aspectos a sua falta de prioridade, já que a doença apresenta caráter crônico e lenta evolução. Além disto, é predominantemente rural, afetando quase sempre populações à margem do processo produtivo e com parcos canais de reivindicação. Os recursos financeiros estão previstos para esta atividade, mas suas aplicações dependem de decisões e competências dos gestores municipais, onde muitas vezes há interesses mais imediatos que a doença de Chagas 18. Segundo Dias 12, a demanda social e política para implementação do controle da doença são mínimos em toda a área endêmica, provavelmente em conseqüência do baixo crédito político da moléstia em termos de visibilidade, já que não são observados efeitos benéficos do programa em curto prazo, diferentemente do que ocorre com epidemias urbanas como o dengue. Percebe-se que está ocorrendo um processo de deterioração de um programa de controle que foi eficiente no passado, arriscando-se a perder alguns resultados alcançados. Tais afirmações foram claramente verificadas em alguns municípios deste estudo, espelhadas na instabilidade dos servidores municipais, na falta de material educativo e na precariedade da manutenção dos veículos 20. Além disso, nossos dados reforçam o proposto por Dias 48 e também sugerido pelos responsáveis técnicos da GRS: quando forem muito baixos os índices de notificação em municípios com histórico de triatomíneos, pesquisas integrais em modelo vertical podem ser desencadeadas a partir da GRS, com fins de avaliação e eventual mudança de percurso do programa local. Logo se faz necessária a coexistência/manutenção da FUNASA (ou reforço substancial nas gerências estaduais ou regionais do PCDCh), com o propósito de supervisionar e dar referência às ações, inclusive mantendo-se reserva de pessoal capacitado a realizar ações supletivas e emergenciais nos municípios que as necessitarem.

Como avaliação positiva da descentralização em termos do PCDCh na região estudada, pode-se apontar o aumento da massa crítica de pessoas com visão na epidemiologia e controle da doença de Chagas nos municípios, constatando-se que em alguns casos estão criadas boas condições municipais para o controle dos vetores desta endemia.

Finalizando, o presente estudo reforça a idéia que a reinfestação de unidades domiciliares por triatomíneos nativos em áreas de vigilância epidemiológica ocorre de maneira continuada e de longa duração, na medida em que existem focos silvestres de algumas espécies vetoras do T. cruzi, carreadoras do flagelado a partir de reservatórios também silvestres. Sabe-se que entre as principais causas desta reinfestação em diversas áreas desinsetizadas está a falta ou descontinuidade da vigilância epidemiológica. Portanto, torna-se urgente e necessário que, nas áreas endêmicas de doença de Chagas sob vigilância, a mesma seja corretamente implantada e adequadamente acompanhada por níveis mais centrais de referência, para se evitar o retorno da endemia humana nestas regiões. 


\section{Resumo}

Atualmente o Programa de Controle da Doença de Chagas (PCDCh) brasileiro está na etapa da vigilância epidemiológica, restando como um dos principais desafios à manutenção e ao aprimoramento desta vigilância. Com o processo de descentralização das ações de saúde, que promoveu a transferência das responsabilidades do controle das endemias para os Estados $e$ municipios, algumas entidades municipais passaram a enfrentar dificuldades na execução do PCDCh visto não terem tradição no controle desta endemia. O presente trabalho objetivou avaliar a situação atual da vigilância epidemiológica da doença de Chagas em 54 municípios da região centro-oeste de Minas Gerais, com vistas à avaliação e ao aprimoramento do processo. No período de julho de 2003 até junho de 2007, foram realizadas 776 capturas, contabilizando 1.390 triatomíneos, provenientes de 37 municípios. A espécie prevalente foi Panstrongylus megistus (99,3\% das capturas). A positividade para flagelados semelhantes ao Trypanosoma cruzi atingiu 8,3\%. A distribuição das capturas mostrou diferenças entre os municípios, e alguns motivos para as mesmas são discutidos no decorrer deste artigo. No conjunto, os dados recomendam fortemente a continuidade e aprimoramento da vigilância epidemiológica na área em questão.

Doença de Chagas; Controle de Doenças Transmissíveis; Vigilância Epidemiológica

\section{Referências}

1. World Health Organization. Control of Chagas disease. Geneva: World Health Organization; 2002.

2. Dias JCP. Controle da doença de Chagas. In: Dias JCP, Coura JR, organizadores. Clínica e terapêutica da doença de Chagas: uma abordagem prática para o clínico geral. Rio de Janeiro: Editora Fiocruz; 1997. p. 453-67.

3. Dias E. Um ensaio de profilaxia na moléstia de Chagas. Rio de Janeiro: Imprensa Nacional; 1945.

4. Vinhaes MC, Dias JCP. Doença de Chagas no Brasil. Cad Saúde Pública 2000; 16 Suppl 2:7-12.

5. Silveira AC, Rezende DF. Epidemiologia e transmissão vetorial da doença de Chagas no Brasil. Rev Soc Bras Med Trop 1994; 27 Suppl 3:11-22.

6. Dias JCP. The impact of Chagas disease control in Latin America: a review. Mem Inst Oswaldo Cruz 2002; 97:603-12.

7. Silveira AC, Vinhaes MC. Doença de Chagas: aspectos epidemiológicos e de controle. Rev Soc Bras Med Trop 1998; 31 Suppl 2:15-60.

\section{Colaboradores}

M. M. Villela e J. C. P. Dias realizaram a elaboração do artigo. J. M. B. Souza e V. P. Mello contribuíram para a redação deste artigo.

\section{Agradecimentos}

Este trabalho é parcialmente subsidiado pelo Programa Especial para Capacitação e Pesquisa em Doenças Tropicais (TDR) [Programa das Nações Unidas para o Desenvolvimento/Banco Mundial/Organização Mundial da Saúde].
8. Carlos ALF, Garcia MHM. O impacto no controle vetorial com inseticida de efeito residual por mais de uma década na área endêmica da doença de Chagas. Rev Soc Bras Med Trop 2005; 38 Suppl 1:39.

9. Schmunis GA. Tripanossomíase americana: seu impacto nas Américas e perspectivas de eliminação. In: Dias JCP, Coura JR, organizadores. Clínica e terapêutica da doença de Chagas: uma abordagem prática para o clínico geral. Rio de Janeiro: Editora Fiocruz; 1997. p. 11-24.

10. Dias JCP. Globalização, iniqüidade e doença de Chagas. Cad Saúde Pública 2007; 23 Suppl 1: S13-22.

11. Moncayo A. Chagas disease: current epidemiological trends after the interruption of vectorial and transfusional transmission in the Southern Cone Countries. Mem Inst Oswaldo Cruz 2003; 98: 577-91. 
12. Dias JCP. Southern Cone Initiative for the elimination of domestic populations of Triatoma infestans and the interruption of transfusional Chagas disease. Historical aspects, present situation, and perspectives. Mem Inst Oswaldo Cruz 2007; 102:11-8.

13. Schofield CJ, Jannin J, Salvatella R. The future of Chagas disease control. Trends Parasitol 2006; 22:583-8.

14. Brasil. Portaria $n^{\circ}$. 1.399. Regulamenta a NOB SUS 01/96 no que se refere às competências da União, estados, municípios e Distrito Federal, na área de epidemiologia e controle de doenças, define a sistemática de financiamento e dá outras providências. Diário Oficial da União 1999; 15 dez.

15. Villela MM, Souza JB, Mello VP, Azeredo BVM, Dias JCP. Vigilância entomológica da doença de Chagas na região centro-oeste de Minas Gerais, Brasil, entre os anos de 2000 e 2003. Cad Saúde Pública $2005 ; 21: 878-86$

16. Brasil. Portaria n ${ }^{\circ}$. 1.172. Regulamenta a NOB SUS 01/96 no que se refere às competências da União, Estados, Municípios e Distrito Federal, na área de Vigilância em Saúde, define a sistemática de financiamento e dá outras providências. Diário Oficial da União 2004, 15 jun.

17. Vasconcelos EM. Educação popular como instrumento de reorientação das estratégias de controle das doenças infecciosas e parasitárias. Cad Saúde Pública 1998; 14 Suppl 2:39-57.

18. Dias JCP. Doença de Chagas: sucessos e desafios. Cad Saúde Pública 2006; 22:2020-1.

19. Ramos Jr. AN, Carvalho DM. Os diferentes significados da certificação conferida ao Brasil como estando livre da doença de Chagas. Cad Saúde Pública 2001; 17:1403-12.

20. Villela MM, Souza JMB, Melo VP, Dias JCP. Vigilância epidemiológica da doença de Chagas em programa descentralizado: avaliação de conhecimentos e práticas de agentes municipalizados em região endêmica de Minas Gerais, Brasil. Cad Saúde Pública 2007; 23:2428-38.

21. Superintendência de Campanhas de Saúde Pública. Manual de normas técnicas da campanha de controle da doença de Chagas. Brasília: Ministério da Saúde; 1980.

22. Vinhaes MC, Schofield C. Trypanosomiasis control: surmouting diminishing returns. Trends Parasitol 2003; 19:112-3.

23. Villela MM, Aleixo A, Souza JMB, Melo VP, Dias JCP. Nota sobre borrifação integral seguida de pesquisa, como estratégia de vigilância epidemiológica da doença de Chagas, em áreas de recrudescência de focos domiciliares de triatomíneos. Rev Soc Bras Med Trop 2006; 39:227-9.

24. Silveira AC. El impacto de la descentralización de los sistemas de salud en la prevención y control de la enfermedad de Chagas: el caso del Brasil. In: Yadón ZE, Gürtler RE, Tobar F, Médici AC, organizadores. Descentralização e gestão do controle das enfermidades transmissíveis na América Latina. Brasília: Organização Pan-Americana da Saúde; 2006. p. 203-14.

25. Cerqueira EM, Assis MMA, Villa TCS, Leite JA. Vigilância epidemiológica no processo de municipalização da saúde em Feira de Santana - BA. Epidemiol Serv Saúde 2003; 12:212-23.
26. Aragão MB. Sobre a dispersão do Triatoma infestans. Rev Soc Bras Med Trop 1971; 5:183-91.

27. Dias JCP, Garcia ALR. Vigilancia epidemiológica con participación comunitaria: un programa de enfermedad de Chagas. Revista Internacional de Educación en Salud 1976; 19:29-44.

28. Dias JCP. Doença de Chagas em Bambuí, Minas Gerais, Brasil. Estudo clínico-epidemiológico a partir da fase aguda entre 1940 e 1982 [Tese de Doutorado]. Belo Horizonte: Universidade Federal de Minas Gerais; 1982.

29. Oliveira Filho AM. New alternatives for the control of triatomines in peridomestic buildings. Rev Soc Bras Med Trop 1989; 22:53-7.

30. Sarquis O, Borges-Pereira J, Cord JRM, Gomes TF, Cabello PH, Lima MM. Epidemiology of Chagas disease in Jaguarana, Ceará, Brazil. I. Presence of triatomines and index of Trypanosoma cruzi infection in four localities of a rural area. Mem Inst Oswaldo Cruz 2004; 99:253-70.

31. Silva RA, Scandar SAS, Pauliquévis-Júnior C Sampaio SMP, Rodrigues VLCC. Ampliação de raio de pesquisa de triatomíneos na atividade de atendimento às notificações em área de Triatoma sordida (Stal, 1859) no Estado de São Paulo. Rev Soc Bras Med Trop 2005; 38:339-43.

32. Forattini OP, Ferreira OA, Rocha-e-Silva O, Rabello EX, Santos JLF. Aspectos ecológicos da tripanossomose americana. II - Distribuição e dispersão local de triatomíneos em ecótopos naturais e artificiais. Rev Saúde Pública 1971; 5:163-91.

33. Diotaiuti L. Ocorrência e biologia do Rhodnius neglectus Lent, 1954, em macaubeiras da periferia de Belo Horizonte, MG [Dissertação de Mestrado]. Belo Horizonte: Universidade Federal de Minas Gerais; 1982.

34. Gürtler RE, Cecere M, Rubel DN, Schweigmann NJ. Determinants of the domiciliary density of Triatoma infestans, vector of Chagas disease. Med Vet Entomol 1992; 6:75-83.

35. Souza RCM, Barbosa SE, Sonoda IV, Azeredo BVM Romanha AJ, Diotaiuti L. Population dynamics of Triatoma vitticeps (Stål, 1859) in Itanhomi, Minas Gerais, Brazil. Mem Inst Oswaldo Cruz 2008; 103:14-20.

36. Diotaiuti L, Faria Filho OF, Carneiro FCF, Dias JCP, Pires HHR, Schofield CJ. Aspectos operacionais do controle do Triatoma brasiliensis. Cad Saúde Pública 2000; 16 Suppl 2:61-7.

37. Dias JCP. Reinfestação do Município de Bambuí por triatomíneos transmissores da doença de Chagas. Mem Inst Oswaldo Cruz 1965; 63:107-19.

38. Silva RA, Scandar SAS, Pauliquévis-Júnior C, Sampaio SMP, Rodrigues VLCC. Ampliação de raio de pesquisa de triatomíneos na atividade de atendimento às notificações em área de Triatoma sordida (Stal, 1859) no Estado de São Paulo. Rev Soc Bras Med Trop 2005; 38:339-43.

39. Falavigna-Guilherme AL, Costa AL, Batista O, Pavanelli GC, Araújo SM. Atividades educativas para o controle de triatomíneos em área de vigilância epidemiológica do Estado do Paraná, Brasil. Cad Saúde Pública 2002; 18:1543-50. 
40. Carcavallo RU, Rocha DS, Girón I, Sherlock IA, Galvão C, Martinez A. Fontes e padrões alimentares. In: Carcavallo RU, Girón GI, Juberg J, Lent $\mathrm{H}$, organizadores. Atlas dos vetores da doença de Chagas nas Américas. Rio de Janeiro: Editora Fiocruz; 1997. p. 537-60.

41. Carcavallo RU, Rodriguez MEF, Salvatella R, Casas SIC, Sherlock IS, Galvão C. Hábitos e fauna relacionada. In: Carcavallo RU, Girón GI, Juberg J, Lent $\mathrm{H}$, organizadores. Atlas dos vetores da doença de Chagas nas Américas. Rio de Janeiro: Editora Fiocruz; 1997. p. 561-600.

42. Gurgel-Gonçalves R, Bezerra W, Cuba CAC. Índice de infestação domiciliar por Rhodnius neglectus (Hemiptera-Reduviidae) capturados nos municípios de Goiás, no período de 2000 a 2005. Rev Soc Bras Med Trop 2006; 39 Suppl 3:158-9.

43. Williams-Blangero S, Vandeberg JL, Teixeira ARL. Attitudes towards Chagas' disease in an endemic Brazilian community. Cad Saúde Pública 1999; 15:7-13.
44. Barretto MP, Carvalheiro JR. Estudos sobre reservatórios e vectores silvestres do Trypanosoma cru$z i$. XII - Inquérito preliminar sobre triatomíneos silvestres no município de Uberaba, Minas Gerais. Rev Bras Biol 1966; 26:5-14.

45. Barretto MP, Albuquerque RDR, Funayama GK. Estudos sobre reservatórios e vectores silvestres do Trypanosoma cruzi. XXXVI - Investigações sobre triatomíneos de palmeiras no município de Uberaba, MG, Brasil. Rev Bras Biol 1969; 29:577-88.

46. Ferraz Filho NA, Rodrigues VLCC. Distribuição e índice de infecção natural de triatomíneos capturados na região de Campinas, São Paulo, Brasil. Rev Soc Bras Med Trop 1987; 20:25-30.

47. Fernandes AJ, Chiari E, Casanova C, Dias JCP, Romanhã AJ. The threat of reintroduction of natural transmission of Chagas disease in Bambuí, Minas Gerais State, Brazil, due to Panstrongylus megistus. Mem Inst Oswaldo Cruz 1992; 87:285-9.

48. Dias JCP. Estratégias e perspectivas atuais da consolidação do controle de vetores na etapa de vigilância epidemiológica. Rev Soc Bras Med Trop 1994; 27 Suppl 2:63-5.

Recebido em 15/Abr/2008

Versão final reapresentada em 05/Ago/2008

Aprovado em 01/Dez/2008 